\title{
Late-onset camptocormia caused by a heterozygous in-frame CAPN3 deletion
}

\author{
Marco Spinazzi ${ }^{\mathrm{a}, \mathrm{g}, *}$, Jerome Poupiot $^{\mathrm{b}, \mathrm{c}}$, Julien Cassereau ${ }^{\mathrm{a}}$, France Leturcq ${ }^{\mathrm{d}}$, Laurent Brunereau ${ }^{\mathrm{e}}$, \\ Edoardo Malfatti ${ }^{\mathrm{f}}$, Isabelle Richard ${ }^{\mathrm{b}, \mathrm{c}}$, Franck Letournel ${ }^{\mathrm{g}}$ \\ a Neuromuscular Reference Center, Department of Neurology, CHU d'Angers, France \\ ${ }^{\mathrm{b}}$ Genethon, 91000, Evry, France \\ ${ }^{\mathrm{c}}$ Université Paris-Saclay, Univ Evry, Inserm, Généthon, Integrare research unit UMR_S951, 91000, Evry-Courcouronnes, France \\ ${ }^{\mathrm{d}}$ Laboratory of Genetics and Molecular Biology, Hôpital Cochin, Paris, France \\ e Radiology Department, CHU de Tours, France \\ ${ }^{\mathrm{f}}$ Neuromuscular Reference Center, Department of Neurology, Hôpital Henri-Mondor Paris, France \\ ${ }^{\mathrm{g}}$ Institute of Neurobiology and Neuropathology CHU d'Angers, France
}

Received 20 May 2020; received in revised form 9 December 2020; accepted 11 February 2021

\begin{abstract}
Camptocormia is defined by a pathological involuntary flexion of the thoracic and lumbar spine that is fully reducible in the supine position. Although originally described as a manifestation of conversion disorder, it is more commonly caused by a wide range of neurological diseases, in particular movement and neuromuscular disorders. We describe here a rare case of late onset camptocormia caused by autosomal dominant calpainopathy due to a heterozygous in-frame deletion in CAPN3 leading to loss of a single lysin amino acid in the catalytic domain of calpain-3. Creatine kinase levels, electromyography, and thigh muscle MRI were normal. Muscle biopsy did not show lobulated fibers and calpain-3 protein expression was not decreased, but in vitro functional assays showed impaired proteolytic function of. Lys $254 \mathrm{del}$ CAPN3. Autosomal dominant calpainopathy should be considered in the differential diagnosis of late onset camptocormia and unexplained paravertebral myopathies even in presence of normal creatine kinase levels, and in absence of lobulated fibers, of decreased calpain-3 protein expression, and of muscle limb involvement.
\end{abstract}

(C) 2021 Elsevier B.V. All rights reserved.

Keywords: Calpainopathy; Calpain-3; Camptocormia; Bent-spine; Paravertebral myopathy.

\section{Introduction}

Camptocormia (from the Greek camptos $=$ bent and cormos $=$ trunk) defines a pathological flexion of the thoracic and lumbar spine that is fully reversible in the supine position and worsens in the standing position or with walking. Since the original description by Brodie in 1818 [1], this term has been widely used during the world wars I and II to describe psychogenic postural manifestations of stress among soldiers, and until few decades ago it has been implicitly linked to conversion disorder. Only in 1992 camptocormia has been used to describe cases of myopathy

\footnotetext{
* Corresponding author.

E-mail address: marco.spinazzi@chu-angers.fr (M. Spinazzi).
}

of paravertebral muscles [2]. In 1999, Djaletti reported camptocormia in eight patients with Parkinson's disease [3], and follow-up studies reported paraspinal myopathy as the structural base of Parkinson's disease camptocormia [4]. It is now recognized that camptocormia comprises a very heterogeneous group of diseases with different mechanisms that ultimately result into paraspinal muscles weakness. The most common etiologies include a wide range of movement and neuromuscular disorders, and degenerative joint diseases, whereas psychogenic camptocormia is nowadays exceedingly rare [5]. Within the neuromuscular etiologies, camptocormia can be caused by diseases affecting the motor unit at any anatomical level, from the motor neuron to the neuromuscular junction and the axial skeletal muscles [6]. It has been recently recognized that axial involvement is very common 
in muscle diseases as part of a more widespread involvement [7]. More rarely, different myopathies can manifest with an exclusive or predominant axial involvement [7-9]. Calpainopathy is a muscular dystrophy affecting the limb girdles most commonly inherited with an autosomal recessive transmission [10], but rare autosomal dominant cases have been recently reported $[11,12]$.

\section{CAPN3 proteolytic activity methods}

L254del CAPN3 cDNA was first generated as a synthetic DNA (Geneart Gene synthesis, Thermo Scientific, Waltham, MA) then cloned into an expressing plasmid under the control of the CMV promoter. WT CAPN3 and the R572P CAPN3 mutant were previously cloned into an expression plasmid [13]. HEK-293 cells were co-transfected with these plasmids, alone or with a reporter plasmid expressing an inactive CAPN3 carrying a mutation in the cysteine of the catalytic site (C129A Substrate) or with the active form of calpain3 (WT). In this construct, the inactive human calpain 3 coding sequence is fused with the fluorescent proteins YFP at the $\mathrm{N}$-Terminus and GFP at the C-terminus. For each transfection, the total quantity of DNA used was $1.8 \mu \mathrm{g}$. Four days after transfection, cell extracts were prepared in RIPA buffer (Thermo Scientific, Waltham, MA) with Protease inhibitor cocktail (Sigma-Aldrich, St. Louis, MO). A BCA protein quantification (Thermo Scientific) was performed to normalize the quantity of protein loaded on the gel. After transfer on a nitrocellulose membrane, a western blot was performed using antibodies against GFP (Rabbit antibody Ab6556, Abcam, dilution 1:500) and Calpain 3 (Mouse antibody NCL-CALP12A2, Leica Biosystems, dilution 1:200 and Goat antibody COP-080049, Operon Biotechnologies, dilution 1:1000).

\section{Case report and CAPN3 proteolytic function analysis results}

A 76-year-old female progressively developed since the age of 67 difficulties in gaining the upright position after bending which she compensated by pushing her hips forward with her hands. This difficulty was increased by bringing small weights or by walking, thus reducing her walking distance to a maximum of $200 \mathrm{~m}$. She complained of frequent cervical and lumbar pain. Her medical history was uneventful and she did not take any drug. In her family, there was no consanguinity. Her father had a waddling gait for which he never underwent any clinical investigation. He died at 98 years following a cerebrovascular accident without significant disability. She had no children, and one brother still alive had no muscular symptoms. Clinical examination revealed a height of $167 \mathrm{~cm}$ for $55 \mathrm{Kg}$. There was a marked kyphosis enhanced by standing and walking, completely abolished in the supine position. Range of motion in spine extension and flexion was normal. Muscle strength was strictly normal except for spine extensors (2/5 MRC), neck flexors (4/5), and hip extensors muscles (3/5). There was no muscular atrophy. Deep tendon reflexes were normal at the upper extremities and reduced at the lower extremities. There was no extrapyramidal rigidity, no bradikynesia, nor tremors. Pulmonary function tests were normal.

Serum creatine kinase levels were normal (136U/L; nv < 145). Normal blood tests included: TSH, lactate, pyruvate, anti-AchR, anti-MUSK antibodies, blood acid maltase activity. EMG was normal. Whole body muscle MRI showed a selective severe symmetrical fatty infiltration of thoracic and lumbar paraspinal muscles, middle and small glutei, without pathological changes at the level of thigh muscles (Fig. 1A).

A left vastus lateralis muscle biopsy showed mildly increased fiber size variability, slight irregularities in the distribution of the oxidative stainings, but absence of necrosis, regenerating fibers, nor lobulated fibers (Fig. 1B). Western blot for dystrophin, dysferlin, sarcoglycans did not show any abnormality. The overall expression of calpain 3 was not reduced, but the ratio between full length 94 KDalton CAPN3, and the cleaved $60 \mathrm{KDa}$ form, was increased in the patient muscle compared to a healthy control and two patients affected by other myopathies unrelated to CAPN3 (Fig. 1C), a finding compatible with altered proteolytic activity. Gene tests for myotonic dystrophy type II (PROMM) and facioscapulohumeral dystrophy type 1 resulted negative.

Next-generation sequencing of 38 genes involved in limb girdle muscular dystrophies (ANO5, CAPN3, CAV3, COL6A1, COL6A2, COL6A3, DAG1, DES, DMD, DNAJB6, DYSF, EMD, FHLI, FKRP, FKTN, GAA, GMPPB, GNE, HNRNPDL, ISPD, LAMA2, LISM2, LMNA, MYOT, PLEC, POMGNT1, POMT1, POMT2, SGCA, SGCB, SGCC, SGCG, TCAP, TNPO3, TRAPPC11, TRIM32, TTN, VCP) showed a heterozygous in-frame deletion in CAPN3 c.759_761del (p.Lys254del). His-brother, who had no neuromuscular complaint and a normal neuromuscular examination, did not carry this CAPN3 variant.

In order to investigate the functional impact of L254del CAPN3 we tested both autolytic and proteolytic activity of L254del CAPN3 in HEK-293 cells, which do not have endogenous expression of CAPN3. WT CAPN3 is able to digest itself (autolysis), as well as other CAPN3 molecules and other substrates (hetero-proteolysis). The control conditions led to the expected results: WT CAPN3 underwent autolysis and R572P CAPN3, a variant known to have impaired proteolytic activity [13], had severely impaired autolysis (Fig. 2A). L254del CAPN3 showed a complete loss of autolytic activity (Fig. 2A). Cotransfection experiments with both the catalytically inactive calpain 3 substrate C129A and L254del CAPN3 or controls, showed that only WT, but not L254del CAPN3 or R572P CAPN3, were capable to proteolyze the C129 substrate, indicating a complete loss of hetero-proteolytic activity in L254del CAPN3 (Fig. 2B). Finally, cotransfection with both WT CAPN3 (WT) and L254del CAPN3 or controls, led to CAPN3 cleavage in all conditions, indicating lack of a dominant negative functional effect of L254del CAPN3 in this condition (Fig. 2C). 
A
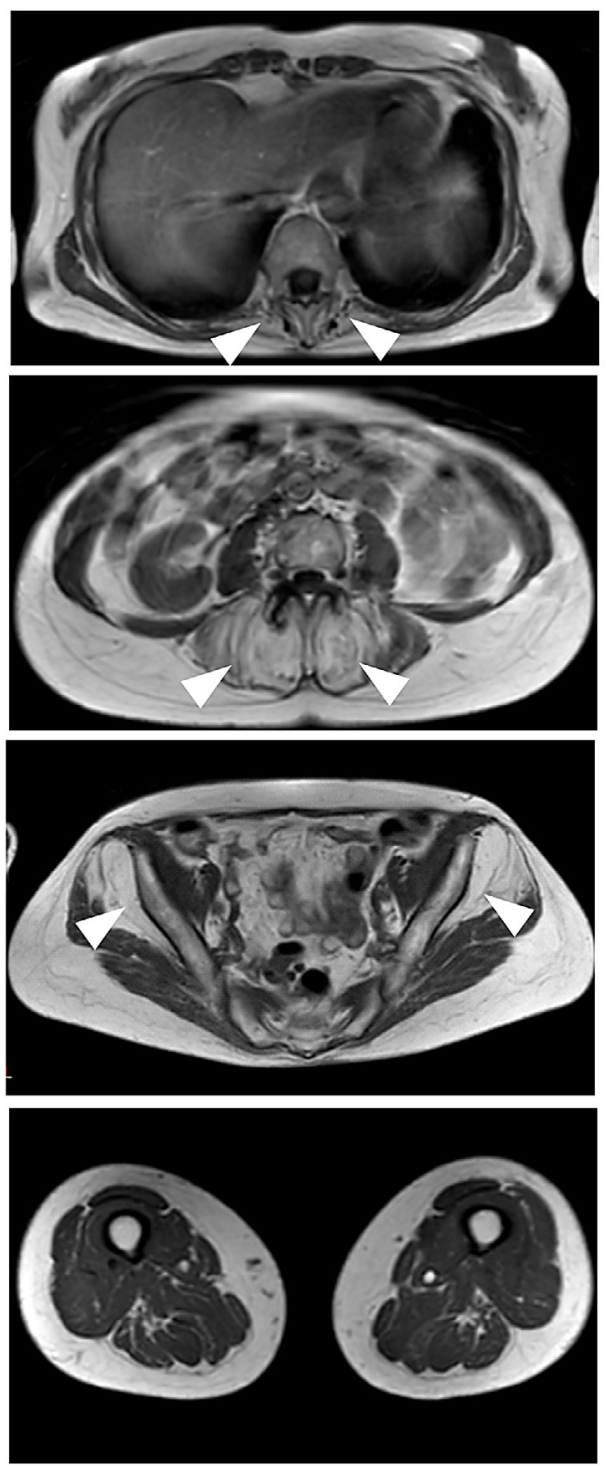

B
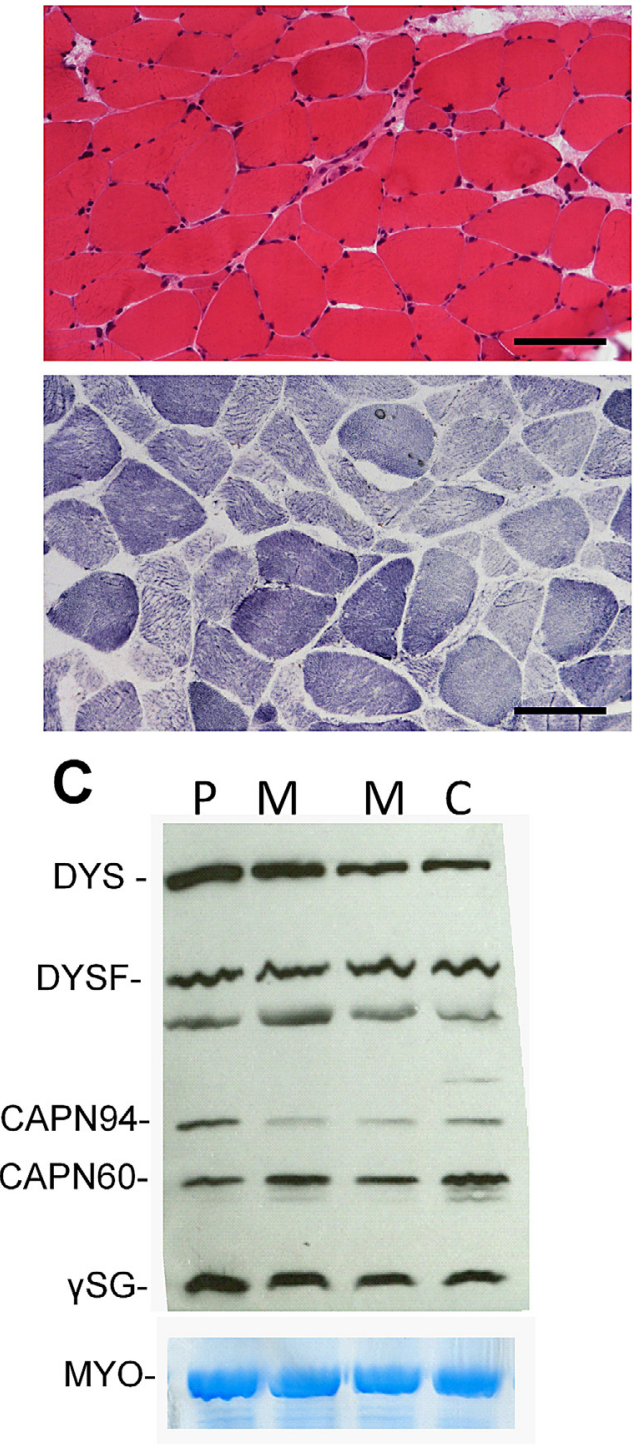

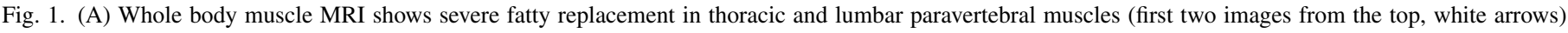

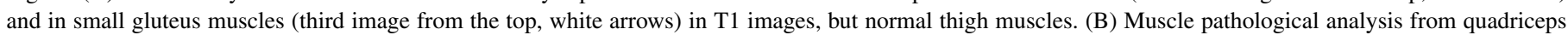

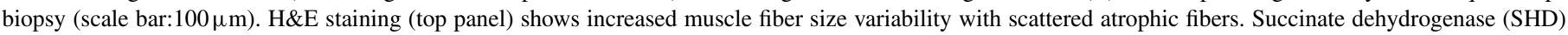

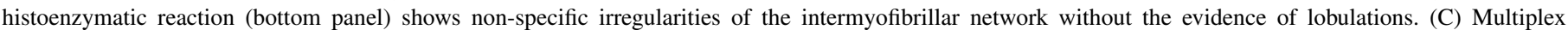

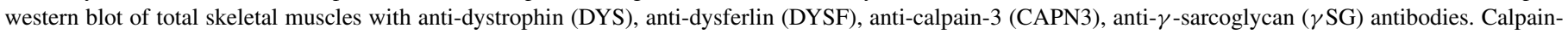

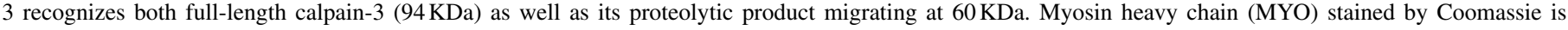
the loading control. $P=$ patient, $M=$ disease controls (patients with myopathies unrelated to $C A P N 3$ ), $C=$ healthy control).

\section{Discussion}

Calpainopathy is among the most common forms of autosomal recessive limb girdle muscular dystrophy worldwide (LGMD2A/LGMDR1). It is caused by mutations in the CAPN3 gene, encoding for a skeletal muscle-specific non lysosomal $\mathrm{Ca}^{2+}$-dependent cysteine protease expressed in the cytosol. Its physiological role is poorly understood. Clinically, it is typically characterized by limb girdle or scapulo-peroneal progressive muscle weakness with variable age at onset between two to forty years [14], with constantly elevated serum creatine kinase. Biochemically, the majority of the mutations are associated with reduced muscle calpain3 levels or impaired autocatalytic activity [15].

More recently, calpainopathies with autosomal dominant forms have been reported in carriers of a single mutated allele, now reclassified as LGMD D4 [16]. The first study of dominant calpainopathy described 37 patients within 10 families carrying a distinct heterozygous 21 base pairs in-frame deletion in the CAPN3 gene (c.643_663del21) [12].

Although this new nosological entity has been initially questioned [17], similar findings have been reported by 

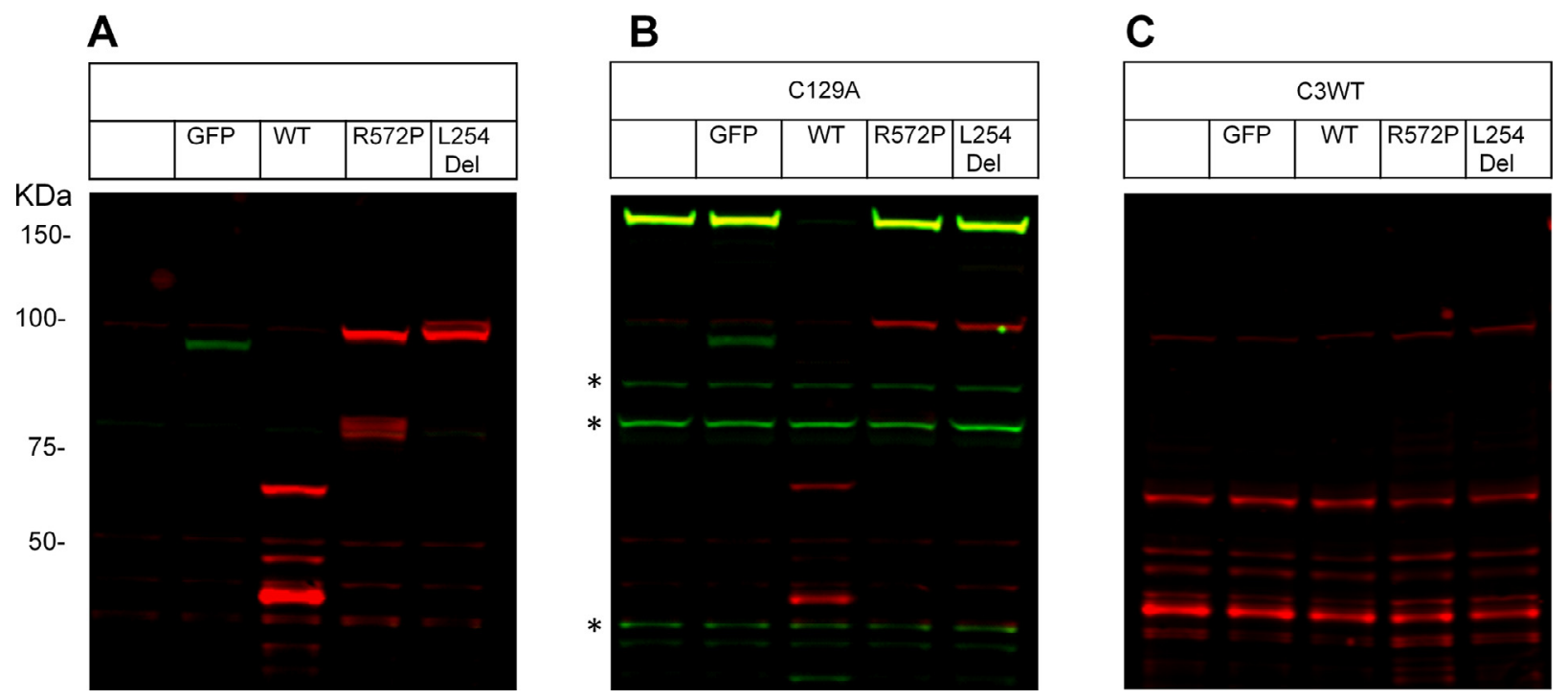

Fig. 2. (A) Autolytic CAPN3 assay. HEK-293 cells were either non transfected or transfected with a control plasmid (GFP), or WT CAPN3 (WT), L254del CAPN3 or the previously described c.1715G $>C$ CAPN3 mutation, leading to R572P CAPN3, as positive control [13]. Cleavage of CAPN3 is revealed by anti CAPN3 antibody (red). The expression of the control plasmid is detected with anti GFP antibody (in green). Only WT CAPN3, but not L254del or R572P are cleaved, indicating the complete loss of autolytic activity of the L254del CAPN3 mutant. (B) Proteolytic CAPN3 assay with catalytic inactive substrate. HEK 293 cells were cotransfected with a control plasmid (GFP), WT CAPN3 (WT) or the two CAPN3 mutants (L254del or the positive control R572P) plus the C129A catalytically inactive CAPN3 substrate fused with YFP at the $\mathrm{N}$ terminus and GFP at the C terminus (C129A, not recognized by anti CAPN3 antibody). Cleavage of the full-length catalytic inactive substrate is revealed by anti GFP (green, at about $150 \mathrm{KDa}$ ). Cleavage of cotransfected WT and the two CAPN3 mutants (L254del or R572P) is revealed by anti CAPN3 antibody (red). Only WT, but not L254del or R572P CAPN3, cleaves the catalytic inactive substrate, indicating loss of proteolytic activity of the L254del CAPN3 deletion. The asterisk indicates nonspecific bands recognized by anti GFP antibody. (C) Proteolytic assay with mutant and catalytically active CAPN3 cotransfection. HEK 293 cells were cotransfected with the control plasmid (GFP), WT CAPN3 (WT) or the two CAPN3 mutants (L254del or R572P) plus WT CAPN3. Cleavage of CAPN3 is revealed by anti CAPN3 antibody (red). Cotransfection of WT and any of the two CAPN3 mutants was not associated with impaired CAPN3 proteolysis indicating absence of dominant functional effect of the L254del CAPN3 deletion.

independent groups reporting additional families carrying the same in-frame deletion [18], and more recently, during the revision of the current article, also missense mutations $[13,19,20]$. These reported cases of dominant calpainopathy are usually characterized by a much milder and later onset phenotype compared to LGMDR1. Patients commonly presented an axial muscular involvement associated with limb weakness, in particular at the level of scapular, gluteal, hamstring and medial gastrocnemius muscles [12,18-20] and CPK levels ranging from normal to markedly elevated.

Late onset camptocormia has been previously reported in a single patient carrying the same in-frame c.759-761delGAA CAPN3 deletion that we report here [11]. Muscle biopsy showed an identical marked fatty infiltration of paravertebral and gluteal muscles as in our case, but with an additional iliopsoas and thigh muscles involvement which was absent in our case.

Unlike our patient, muscle biopsy showed a marked presence of lobulated fibers, a common but incompletely sensitive and specific histological finding in calpainopathy [11]. The authors strongly suspected but could not conclude a diagnosis of a dominant form of calpainopathy based on a single observation. This deletion, already described as pathogenic in some autosomal recessive cases, seems to be relatively rare since it has been reported in about $3.7 / 100^{\prime} 000$ exomes in the European population in the gnomAD database in dbSNP (rs794727697). Our independent observation supports the c.759-761delGAA CAPN3 heterozygous deletion as the cause of the reported late-onset camptocormia.

The molecular mechanism underlying autosomal dominant calpainopathy is unclear. Severe reduction in CAPN3 protein expression has been observed in most [12,20] but not all cases with dominant calpainopathy [19] leading to hypothesize a possible dominant negative molecular mechanism on protein stability, possibly via polymerization of WT and deleted calpain-3 in dimers [12] or homo-trimers [21]. In our case the overall expression of CAPN3 was not reduced, arguing against a dominant negative effect on CAPN3 protein stability. Instead, we noticed an increased ratio of full length uncleaved CAPN3 over the cleaved form compared to normal or disease controls, suggesting partially impaired catalytic activity [22], which we expected since L254del leads to a loss of a lysine in catalytic domain of CAPN3. This hypothesis was further confirmed in our cell-based proteolytic assays that indicate a loss of autolytic and hetero-proteolytic function of L254del CAPN3, but no dominant negative functional effect. These data suggest that in our case the molecular mechanism is likely due to haploinsufficiency. Impaired proteolytic activity has been recently reported in two other $C A P N 3$ variants associated with dominant calpainopathy in which functional experiments have been conducted [13,19]. Although we cannot rule out that non-enzymatic functions 
of CAPN3 are involved we believe that decreased CAPN3 proteolytic activity, likely play an important role in the pathogenesis of dominant calpainopathy.

Dominant calpainopathy seems to be associated with variable age of onset comprised between 15 and 84 years of age [12,18,19], and severity ranging from asymptomatic to severely affected patients. As in other genetic diseases the underlying reason for this phenotypic variability and incomplete penetrance is unknown. The D4Z4 repeat contraction associated with facio-scapulohumeral dystrophy has been found to be present in as many as 3\% healthy individuals while the prevalence of this disease occurs only in about $1 / 20^{\prime} 000$ [23]. Therefore, in dominant calpainopathy, as well as in facioscapulo-humeral muscular dystrophy and other genetic diseases, additional genetic, epigenetic, and likely also nongenetic factors are involved and deserve to be investigated further.

The pattern of muscle involvement is also variable in dominant calpainopathy, and is typically characterized by limb girdle and axial muscles [12,18-20]. Camptormia has been reported in few other cases of dominant calpainopathy, but, unlike our case, associated with thigh muscles involvement $[11,19]$. The reasons for the frequent paravertebral muscle involvement is not known, but paravertebral muscles are more susceptible than leg muscles to age-related degeneration [24], perhaps due to their prolonged activation with significant anti-gravity workload. We believe that heterozygous CAPN3 mutations may be an under-recognized cause of camptocormia and age-related axial muscle weakness. We speculate whether single allele mutations in CAPN3 may constitute a risk factor to develop paravertebral myopathy during aging. Conceptually, a similar genetic mechanism has been observed in carriers of mutations in $G B A$, encoding for the enzyme galactocerebrosidase. Homozygous or compound heterozygous mutations cause the lysosomal-storage disease Gaucher disease, while heterozygous mutations confer a 20fold increased risk to develop Parkinson's disease by the age of 80 [25]. Future studies are warranted to investigate the prevalence of CAPN3 mutations in patients with unexplained camptocormia and of paraspinal myopathy in heterozygous carriers of CAPN3 mutations.

In conclusion, our observations support a pathogenic role of the c.759_761del CAPN3 deletion as one possible cause of camptocormia by a haploinsufficiency mechanism. CAPN3 gene mutations should be considered in the differential diagnosis of unexplained late-onset camptocormia and axial myopathies even in absence of lobulated fibers, normal creatine kinase levels and absence of decreased CAPN3 expression and thigh muscles involvement. Increased ratio between full length and cleaved CAPN3 can be a hint for impaired CAPN3 proteolytic activity warranting further investigation.

\section{Declaration of Competing Interest}

None.

\section{Acknowledgments}

MS is supported by AFM Telethon, France (23019).

\section{References}

[1] Brodie B. Pathological and surgical observations on the diseases of the joints. London: Longman; 1818.

[2] Hilliquin P, Menkès CJ, Laoussadi S, J-D C, S G. Camptocormia in the elderly. A new entity by paravertebral muscle involvement? Rev Rhum Mal Osteoartic 1992;59:169-75.

[3] Djaldetti R, Mosberg-Galili R, Sroka H, Merims D, Melamed E. Camptocormia (Bent spine) in patients with Parkinson's disease Characterization and possible pathogenesis of an unusual phenomenon. Mov Disord 1999;14:443-7.

[4] Margraf NG, Wrede A, Rohr A, Schulz-Schaeffer WJ, Raethjen J, Eymess A, et al. Camptocormia in idiopathic Parkinson's disease: A focal myopathy of the paravertebral muscles. Mov Disord 2010;25:542-51.

[5] Ali F, Matsumoto JY, Hassan A. Camptocormia: Etiology, diagnosis, and treatment response. Neurol Clin Pract 2018;8:240-8.

[6] Margraf NG, Wrede A, Deuschl G, Schulz-schaeffer WJ. Pathophysiological Concepts and Treatment of Camptocormia. J Parkinsons Dis 2016;6:485-501. doi:10.3233/JPD-160836.2016;6: 485-501.

[7] Witting N, Andersen LK, Vissing J. Axial myopathy: An overlooked feature of muscle diseases. Brain 2016;139:13-22.

[8] Ghosh PS, Milone M. Camptocormia as presenting manifestation of a spectrum of myopathic disorders. Muscle and Nerve 2015;52:1008-12.

[9] Laroche M, Cintas P. Bent spine syndrome (camptocormia): A retrospective study of 63 patients. Joint Bone Spine 2010;77:593-6.

[10] Richard I, Broux O, Allamand V, Fougerousse F, Chiannilkulchai N, Bourg N, et al. Mutations in the proteolytic enzyme calpain 3 cause limb-girdle muscular dystrophy type 2A. Cell 1995;81:27-40.

[11] Liewluck T, Goodman BP. Late-onset axial myopathy and camptocormia in a calpainopathy carrier. J Clin Neuromuscul Dis 2012;13:209-13.

[12] Vissing J, Barresi R, Witting N, Van Ghelue M, Gammelgaard L, Bindoff LA, et al. A heterozygous 21-bp deletion in CAPN3 causes dominantly inherited limb girdle muscular dystrophy. Brain 2016;139:2154-63.

[13] Vissing J, Dahlqvist JR, Roudaut C, Poupiot J, Richard I, Duno M, et al. A single c. $1715 \mathrm{G}>\mathrm{C}$ calpain 3 gene variant causes dominant calpainopathy with loss of calpain 3 expression and activity. Hum Mutat 2020;41:1507-13. doi:10.1002/humu.24066.

[14] Angelini C, Fanin M, et al. Calpainopathy. In: Adam MP, Ardinger HH, Pagon RA, et al., editors. GeneReviews® [Internet]. Seattle (WA): University of Washington, Seattle; 2005 May 10. p. 1993-2021. Available from: https://www.ncbi.nlm.nih.gov/books/NBK1313/

[15] Fanin M, Fulizio L, Nascimbeni AC, Spinazzi M, Piluso G, Ventriglia VM, et al. Molecular diagnosis in LGMD2A: Mutation analysis of protein testing? Hum Mutat 2004;24:52-62.

[16] Straub V, Murphy A, Udd B. 229th ENMC international workshop: Limb girdle muscular dystrophies - Nomenclature and reformed classification Naarden, the Netherlands, 17-19 March 2017. Neuromuscul Disord 2018;28:702-10. doi:10.1016/j.nmd.2018.05.007.

[17] Sáenz A, De Munain AL. Dominant LGMD2A: Alternative diagnosis or hidden digenism? Brain 2017;140:e7. doi:10.1093/brain/aww281.

[18] Martinez-Thompson JM, Niu Z, Tracy J, Moore S, Swenson A, Wiebe E, Milone M. Autosomal dominant calpainopathy due to heterozygous CAPN3 C.643_663del21. Muscle Nerve 2018;57:679-83.

[19] Cerino M, Campana-Salort E, Salvi A, Cintas P, Renard D, Juntas Morales R, et al. Novel CAPN3 variant associated with an autosomal dominant calpainopathy. Neuropathol Appl Neurobiol 2020;46:564-78. doi:10.1111/nan.12624. 
[20] González-Mera L, Ravenscroft G, Cabrera-Serrano M, Ermolova N, Domínguez-González C, Arteche-López A, et al. Heterozygous CAPN3 missense variants causing autosomal-dominant calpainopathy in seven unrelated families. Neuropathol Appl Neurobiol 2020. doi:10.1111/nan. 12663.

[21] Hata S, Doi N, Shinkai-ouchi F, Ono Y. A muscle-specific calpain, CAPN3, forms a homotrimer. Biochim Biophys Acta Proteins Proteom 2020;1868:140411. doi:10.1016/j.bbapap.2020.140411.

[22] Groen EJ, Charlton R, Barresi R, Anderson LV, Eagle M, Hudson J, et al. Analysis of the UK diagnostic strategy for limb girdle muscular dystrophy 2A. Brain 2007;130:3237-49. doi:10.1093/brain/awm259.
[23] Scionti I, Fabbri G, Fiorillo C, Ricci G, Greco F, D'Amico R, et al. Facioscapulohumeral muscular dystrophy: New insights from compound heterozygotes and implication for prenatal genetic counselling. J Med Genet 2012;49:171-8. doi:10.1136/jmedgenet-2011-100454.

[24] Dahlqvist JR, Vissing CR, Hedermann G, Thomsen C, Vissing J. Fat Replacement of Paraspinal Muscles with Aging in Healthy Adults. Med Sci Sports Exerc 2017;49:595-601.

[25] Regan GO, Balestrino R, Schapira AH. Glucocerebrosidase Mutations in Parkinson Disease. J Parkinsons Dis 2017;7:411-22. doi:10.3233/ JPD-171092. 